UMD-PP-95-32

\title{
Anomalous Magnetic Moment of Anyons
}

\author{
Gil Gat ${ }^{1}$ and Rashmi Ray ${ }^{2}$ \\ Physics Department \\ University of Maryland \\ College Park, MD 20742 \\ U.S.A
}

\begin{abstract}
The anomalous magnetic moment of anyons is calculated to leading order in a $1 / N$ expansion. It is shown that the gyromagnetic ratio g remains 2 to the leading order in $1 / N$. This result strongly supports that obtained in [2], namely that $\mathrm{g}=2$ is in fact exact.
\end{abstract}

${ }^{1}$ ggat@delphi.umd.edu

${ }^{2}$ rray@delphi.umd.edu 


\section{Introduction}

In two spatial dimensions there is the intriguing possibility of anyons, particles with arbitrary spin and statistics [5]. Although there is currently no direct experimental evidence for the existence of anyons they may exist as quasi- particles in realistic systems like the fractional quantum Hall system.

A consistent second quantized theory of anyons where the particle operators create and destroy anyons is yet to be formulated in a satisfactory way. In view of this, most analyses are performed with a theory of fermions (or bosons) coupled minimally to a gauge field whose action contains a Chern-Simons (C-S) term. The C-S term, which provides topological mass for the gauge field, transmutes the fermion(or boson) spin and statistics to those appropriate for anyons.

A matter of vital importance is the investigation of the electromagnetic interaction of anyons, in particular of the gyromagnetic ratio $(\mathrm{g})$. It has been demonstrated in [1] that starting from a fermionic (bosonic) theory the anomalous magnetic moment of anyons (at the one loop level) is proportional to the induced fractional spin , with a gyromagnetic ratio of 2. Subsequent work [2] tends to suggest that this ratio remains 2 to all orders i.e. it is exact.

It is well known that the fractional spin is unrenormalized beyond one loop (ColemanHill theorem [3]). Thus for the g-factor to remain 2 to all orders in perturbation theory the anomalous magnetic moment ought to be unrenormalized beyond one loop too. One might try to extract this information from an analysis of the entire weak coupling expansion. It is not clear to us however what symmetry if any will facilitate this task . Instead we have espoused in this work a more modest approach, namely the $1 / \mathrm{N}$ expansion. The $1 / \mathrm{N}$ expansion effectively resums an infinite subclass of diagrams (i.e. the bubble diagrams). It thus contains information beyond weak coupling expansion and at least in the infinite $\mathrm{N}$ limit it becomes exact. Except for lattice simulations, this method is 
possibly the only systematic non-perturbative method one can use.

\section{The Model}

Consider a model with $2 N+1$ identical fermionic species coupled minimally to a gauge field in $2+1$ dimensions

$$
\mathcal{L}_{E}=\bar{\psi}_{a}\left(i \not \partial+m-\frac{e}{\sqrt{N}} \not\right) \psi_{a}-\frac{1}{4} F_{\mu \nu} F^{\mu \nu}-i \frac{\kappa}{8 \pi} \epsilon^{\mu \nu \rho} A_{\mu} \partial_{\nu} A_{\rho}
$$

An odd number of fermion flavors is necessary to satisfy the requirements of $\mathrm{P}$ and $\mathrm{T}$ violation. This choice of lagrangian is quite general since as we shall see both a C-S term and a Maxwell term would have been generated radiatively even if our original lagrangian did not contain them.

In order to obtain a sensible expansion in powers of $1 / N$, we fix $e^{2} N=\gamma$ and reexpress the Lagrangian in eq.(1) in terms of $\gamma$. The generating functional for the Green functions of the theory is

$$
\mathcal{Z}=\int D \bar{\psi} D \psi D A \delta(\partial \cdot A) e^{-S_{A}-S_{f}}
$$

where

$$
\begin{aligned}
S_{A} & =-\int d^{3} x\left[\frac{1}{4} F_{\mu \nu} F^{\mu \nu}+i \frac{\kappa}{8 \pi} \epsilon^{\mu \nu \rho} A_{\mu} \partial_{\nu} A_{\rho}\right] \\
S_{f} & =\int d^{3} x \bar{\psi}_{a}\left(i \not \partial+m-\frac{\gamma}{\sqrt{N}} \not A\right) \psi_{a}
\end{aligned}
$$

Integrating over the fermionic fields we get

$$
\begin{aligned}
\mathcal{Z} & =\int D A \delta(\partial \cdot A) e^{-S_{A}-W_{f}} \\
W_{f} & =N \operatorname{Tr} \log (i \not \partial+m)+N \operatorname{Tr} \sum_{n=1}^{\infty} \frac{1}{n}\left(\frac{1}{\sqrt{N}}\right)^{n}\left(\frac{1}{i \not \partial+m} \not A\right)^{n}
\end{aligned}
$$

The lowest order term in $W_{f}$, after discarding the field independent term, vanishes on using any soft cut-off procedure that respects gauge invariance (e.g. dimensional regularization). The quadratic term in the field gives the leading order improved inverse 
propagator for the gauge field inverse propagator for the gauge field (in the Landau gauge)

$$
D(p)_{\mu \nu}=D(p)_{\mu \nu}^{\text {tree }}-\Pi(p)_{\mu \nu}
$$

where $D(p)^{\text {tree }}$ is given by $D(p)^{\text {tree }}=-p^{2}\left[\mathcal{P}_{\mu \nu}+i \frac{\kappa}{4 \pi} \epsilon_{\mu \nu \rho} \frac{p^{\rho}}{p^{2}}\right]$ and

$$
\Pi_{\mu \nu}=i \int \frac{d^{3} k}{(2 \pi)^{3}} \frac{\operatorname{tr}\left(\gamma_{\mu}(\not k+m) \gamma_{\nu}(\not k-\not p+m)\right)}{\left[(k-p)^{2}-m^{2}\right]\left[k^{2}-m^{2}\right]}
$$

the $2+1$ dimensional Dirac matrices satisfy

$$
\gamma_{\mu} \gamma_{\nu}=g_{\mu \nu}-i \epsilon_{\mu \nu \rho} \gamma^{\rho} \quad \operatorname{tr}\left(\gamma_{\mu} \gamma_{\nu}\right)=2 g_{\mu \nu}
$$

Using dimensional regularization and standard Feynman parameters we find

$$
\Pi_{\mu \nu}(p)=F(p) \mathcal{P}_{\mu \nu}+i m \epsilon_{\mu \nu \rho} \frac{p^{\rho}}{p^{2}} G(p)
$$

where

$$
\begin{aligned}
\mathcal{P}_{\mu \nu} & =g_{\mu \nu}-\frac{p_{\mu} p_{\nu}}{p^{2}} \\
G(p) & =-\frac{p}{4 \pi} \log \left(\frac{p+2 m}{p-2 m}\right)-\frac{i p}{4} \\
F(p) & =\frac{1}{4 \pi}\left[m-\frac{p^{2}+4 m^{2}}{4 p} \log \left(\frac{p+2 m}{p-2 m}\right)-\frac{i \pi\left(p^{2}+4 m^{2}\right)}{4 p}\right]
\end{aligned}
$$

Combining eq.(5) and (\$) for $D(p)_{\mu \nu}$ and inverting we get the leading order gauge propagator

$$
G(p)_{\mu \nu}=-i \frac{\left(-p^{2}\right) \Pi_{e}}{\Pi_{e}^{2} p^{2}-\Pi_{o}^{2} M^{2}}\left[\mathcal{P}_{\mu \nu}-i M \frac{\Pi_{o}}{\Pi_{e}} \epsilon_{\mu \nu \rho} \frac{p^{\rho}}{p^{2}}\right]
$$

where $\Pi_{e}=p^{2}+F(p)$ and $\Pi_{o}=p^{2}+G(p)$ are the even and odd parts of the inverse propagator [4].

For large enough $M=\frac{\kappa}{4 \pi}$ i.e. the anyons spin $S=\frac{1}{2}+\frac{1}{\kappa}$ is very close to $1 / 2$. Thus as has been discussed in [6], an anyon may be viewed as a point charge surrounded by a gauge field cloud of size $\sim \frac{1}{M}$. Thus in the limit $M \gg m$, the cloud collapses on to the charge and such a system of charge-cloud composites will display fractional statistics 
upon interchange even for arbitrarily small separation between the particles円 Thus such a limit as envisioned above seems to be indicated for a system of anyons.

\section{Anomalous Magnetic Moment}

The anomalous magnetic moment of fermions is defined by using the Gordon decomposition of the fermionic vector current. In $2+1$ dimensions it has the following form:

$$
\bar{\psi}(p+q) \gamma_{\mu} \psi(p)=\frac{(2 p+q)_{\mu}}{2 m} \bar{\psi}(p+q) \psi(p)+i \epsilon_{\mu \nu \lambda} \frac{q^{\lambda}}{2 m} \bar{\psi}(p+q) \gamma^{\nu} \psi(p) .
$$

By coupling this current to an external electromagnetic field it is easy to see that the magnetic moment is the coefficient of $i \epsilon_{\mu \nu \lambda} \frac{p^{\nu} q^{\lambda}}{m}$. The magnetic moment $\mu$ can be written as $\mu=\frac{g}{2 m} S=\frac{1}{2 m}$.

For fermions the spin $S=1 / 2$ so the gyromagnetic ratio $g=2$. For anyons $S=\frac{1}{2}+\frac{1}{\kappa}$ does not shift from its value which is determined at one loop [1]. It was shown that to this order $g$ is the same as for fermions i.e. $g=2$. Therefore if $\mu$ gets any correction it must alter the gyromagnetic ratio. In what follows we give the details of a calculation of $\mu$ to leading order in $1 / N$. This calculation goes beyond one loop because the leading order of $1 / N$ already sums an infinite subset of weak coupling diagrams. In fig.1 we show the leading order diagrams that contribute to the anomalous magnetic moment. In addition to the usual weak coupling diagram $(a)$ we have a two loop diagram $(b)$ that is of the same order $\left(\frac{1}{\sqrt{N}}\right)^{3 / 2}$, however this diagram vanishes identically due to $C$ invariance. We thus consider only diagrams $(a)$.

$$
\Gamma_{a}(p, q)_{\mu}=-i \int \frac{d^{3} k}{\left(2 \pi^{3}\right)} \frac{\bar{u}(p-q) \gamma_{\nu}(\not p-\not q-k+m) \gamma_{\mu}(\not p-\not k+m) \gamma_{\lambda} u(p)}{\left[(p-k)^{2}-m^{2}\right]\left[(p-k-q)^{2}-m^{2}\right]} G(k)^{\nu \lambda}
$$

The large $N$ gauge field propagator in eq. (5) has a very complicated form. In particular this form does not render itself for Feynman parametrization. It is therefore

\footnotetext{
${ }^{1}$ Actually for large enough $M \Pi_{e}^{2} p^{2}-\Pi_{o} M^{2}$ develops an imaginary part, so that the gauge field becomes unstable [7], however the width of this resonance is very small and goes as $1 / M$
} 
useful to use the Källen- Lehmann representation for this propagator.

$$
G(p)_{\mu \nu}=-i\left[\int_{0}^{\infty} d s \frac{\rho_{1}(s)}{p^{2}-s^{2}+i \epsilon} \mathcal{P}_{\mu \nu}(p)-i M \epsilon_{\mu \nu \rho} \frac{p^{\rho}}{p^{2}} \int_{0}^{\infty} d s \frac{\rho_{2}(s)}{p^{2}-s^{2}+i \epsilon}\right]
$$

where

$$
\rho_{1}(s)=\frac{s}{\pi} \operatorname{Im} \frac{\Pi_{e}(s)}{\Pi_{e}(s)^{2} s-\Pi_{o}(s)^{2} M^{2}} \quad \rho_{2}(s)=\frac{s}{\pi} \operatorname{Im} \frac{\Pi_{o}(s)}{\Pi_{e}(s)^{2} s-\Pi_{o}(s)^{2} M^{2}}
$$

In this form the propagator is a superposition of free propagators where the mass squared has been replaces by $s$. We can therefore essentially do all calculations with propagators that have the free form, keeping in mind that we should integrate the end result over $s$ with a weight function $\rho_{1}(s)$ or $\rho_{2}(s)$ (Fig.2).

Going back to the calculation we can use the natural split of the propagator eq.(5) to $\mathrm{P}$-even and $\mathrm{P}$-odd parts to rewrite $\Gamma_{a}(p, q)_{\mu}=\Gamma_{a}(p, q)^{\text {odd }}+\Gamma_{a}(p, q)^{\text {even }}$. As we are interested in the anomalous magnetic moment we shall look at the P-odd part of $\Gamma_{a}(p)_{\mu}$. Using $(\not p-m) u(p)=\bar{u}(p-q)(\not p-\not q-m)=0$ we get after some algebra :

$$
\Gamma_{a}^{o d d}=e^{2} M \epsilon_{\lambda \nu \sigma} \int_{0}^{\infty} d s \rho_{2}(s) \int \frac{d^{3} k}{(2 \pi)^{3}} k_{\sigma} \frac{\bar{u}(p-q)\left[2(p-q)_{\nu}+\not k \gamma_{\nu}\right] \gamma_{\mu}\left[2 p_{\lambda}+\gamma_{\lambda} \not k\right] u(p)}{k^{2}\left[k^{2}-s\right]\left[(p-k)^{2}-m^{2}\right]\left[(p-k-q)^{2}-m^{2}\right]}
$$

from the $\gamma$ matrix identities eq.(7) We can write $\Gamma_{a}^{o d d}=\Gamma_{a}^{(1)}+\Gamma_{a}^{(2)}$ Where

$$
\begin{aligned}
\Gamma_{a}^{(1)} & =2 e^{2} M \epsilon_{\lambda \nu \sigma} \int_{0}^{\infty} \frac{\rho_{2}(s)}{s} \Gamma_{a}^{(1)}(s) \\
\Gamma_{a}^{(1)}(s) & =\frac{1}{\sqrt{s}} \int k_{\sigma} \frac{p_{\lambda} \not k \gamma_{\nu} \gamma_{\mu}+(p-q)_{\nu} \gamma_{\mu} \gamma_{\lambda} \not k}{\left[(p-k-q)^{2}-m^{2}\right]\left[(p-k)^{2}-m^{2}\right]}\left[\frac{1}{k^{2}}-\frac{1}{k^{2}-s}\right] \\
\Gamma_{a}^{(2)} & =e^{2} M \int_{0}^{\infty} \rho_{2}(s) \Gamma_{a}^{(2)}(s) \\
\Gamma_{a}^{(2)}(S) & =\int \frac{d^{3} k}{(2 \pi)^{3}} \frac{\epsilon^{\sigma \lambda \mu} \gamma^{\lambda} k^{\sigma}+k_{\mu}+\not k \gamma_{\mu}}{\left[(p-k-q)^{2}-m^{2}\right]\left[(p-k)^{2}-m^{2}\right]\left[k^{2}-s\right]} .
\end{aligned}
$$

using standard Feynman parametrization we can perform the integration over $k$. To simplify the calculation we also assume that $q \ll M, m$ and use the on shell condition $p^{2}=m^{2}$. Isolating the term that is proportional to $\epsilon_{\mu \nu \rho} p^{\mu} q^{\rho}$ we get

$$
\begin{aligned}
\mu_{a} & =-\frac{e^{2} M}{m^{5}} \int_{o}^{\infty} d s \frac{\rho_{2}(s)}{s}\left[6 m \sqrt{s}+8 m^{2}+(3 s+6 \sqrt{s} m) \log \left[\frac{2 m \sqrt{s}-s}{4 m^{2}-s}\right]\right] \frac{\sqrt{s}}{\sqrt{s}+2 m} \\
& =\frac{1}{m \kappa}
\end{aligned}
$$


we have calculated this integral numerically. In the limit $\kappa \rightarrow \infty$ the corrections to the value obtained in weak coupling i.e $\mu=\frac{1}{m}\left(\frac{1}{2}+\frac{1}{\kappa}\right)$ are down by power of $\frac{1}{\kappa}$. Thus for large $\kappa$ there is no difference between the weak coupling result and the large $\mathrm{N}$ result even though the later uses an improved propagator for the gauge field.

\section{Conclusions}

In this paper we have shown that for $P$ and $T$ violating Q.E.D in the anyonic limit the gyromagnetic ratio of anyons is exactly 2 to leading order in $1 / N$. By using the large $N$ method we have furnished a strong non-perturbative support to the result claimed in [2].

\section{Acknowledgements}

We thank V.P. Nair for suggesting this problem, A. Kovner for a useful discussion and J. Simon for helping us with the graphics. This work is partially supported by the National Sience Foundation.

\section{References}

[1] I. Kogan and G. Semenoff Nuc. Phys. B 368, 718 (1992)

[2] V. P. Nair, A. P. Polychronakos and C. Chou Phys. Lett B 304, 105 (1993)

[3] S. Coleman and B. Hill, Phys. Lett B 159, 184, (1985)

[4] S. Deser, R. Jackiw and S. Templeton, Ann. Phys 140, 372, (1982)

[5] F. Wilczek, "Fractional statistics and anyon superconductivity", (World Scientific 1990)

[6] K. Shizuya and H. Tamura, Phys. Lett B 252, 412 (1992)

[7] L. Brown, Quantum Field Theory, (Cambridge University Press ,1992) 


\section{Figure Captions}

Fig.1 The diagrams contributing to the anomalous magnetic moment in the leading order in $1 / \mathrm{N}$.

Fig. $2 \rho_{1}(p)$ and $\rho_{2}(p)$ for $M=4, \frac{e^{2}}{4 \pi}=0.3$ and $m=1$ 

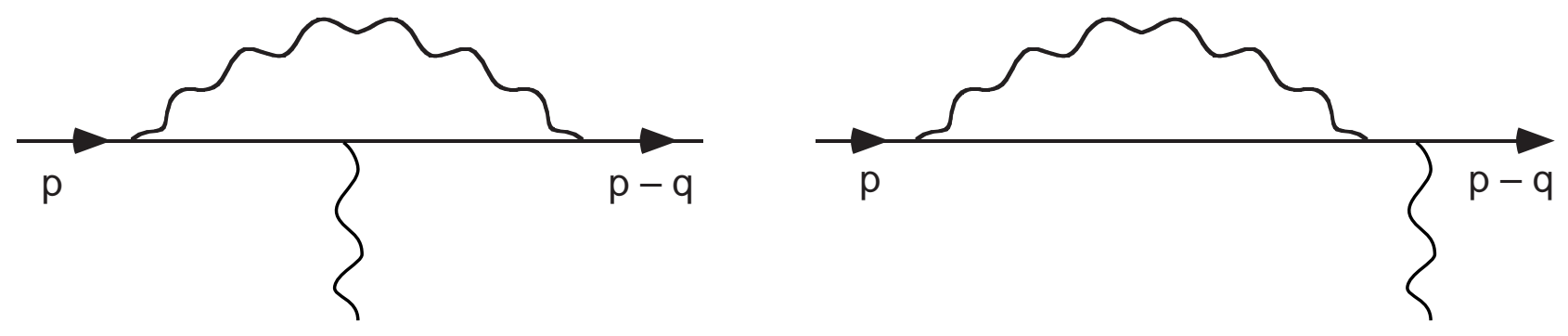

a

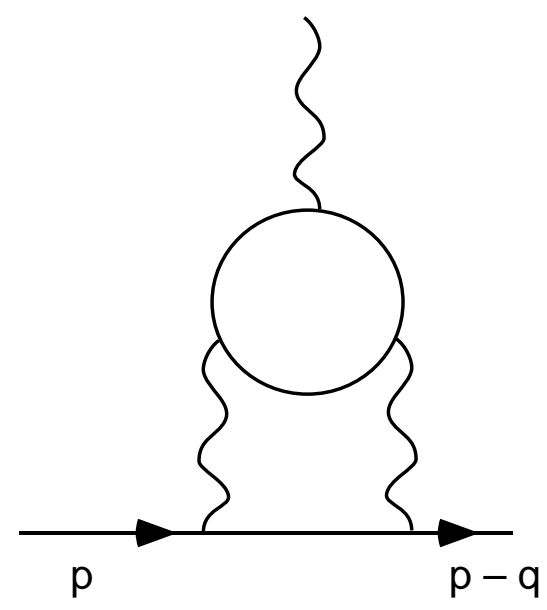

b

Figure 2 


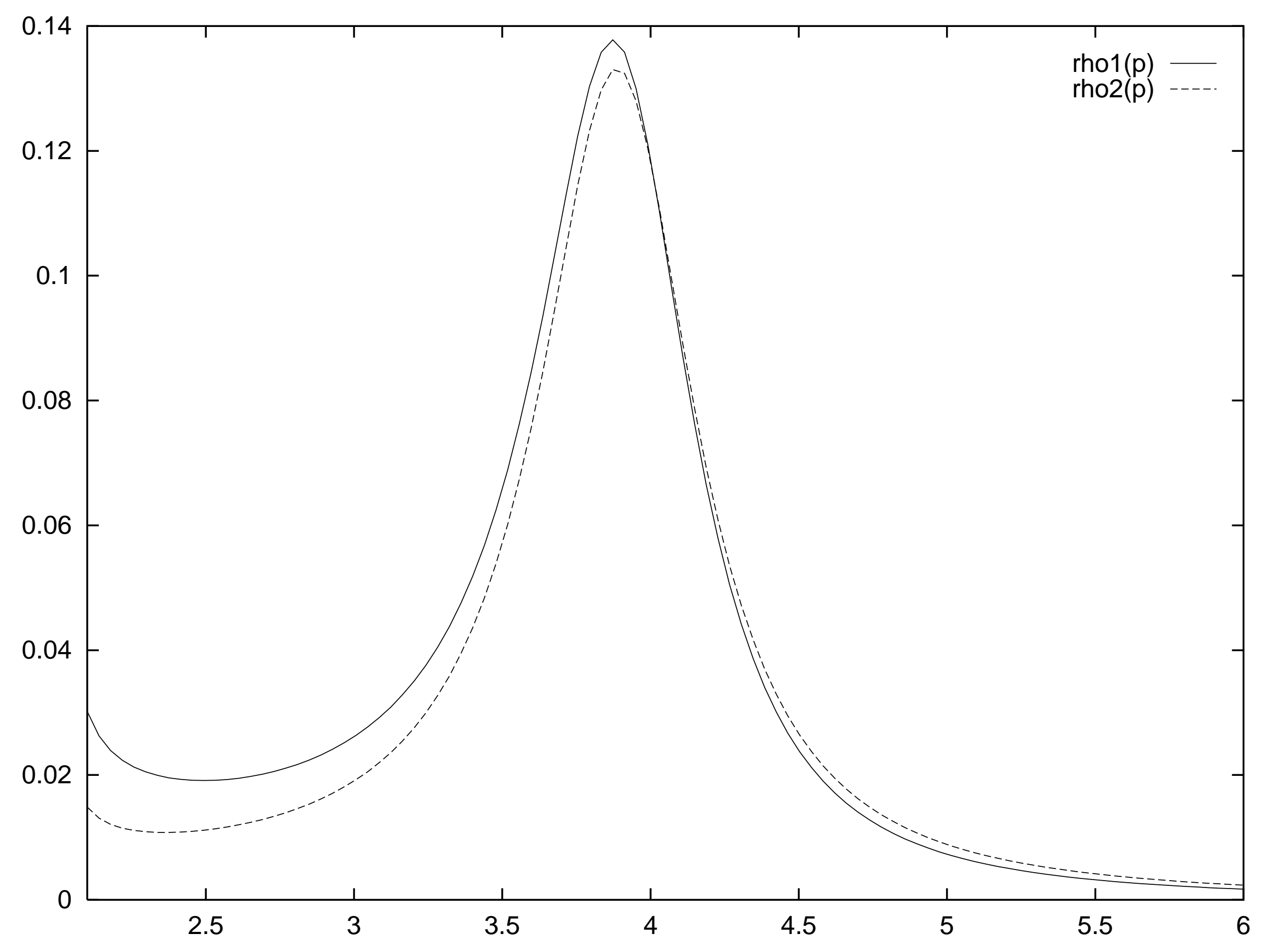

